\title{
Bufalin enhances the antitumor effect of gemcitabine in pancreatic cancer
}

\author{
YING CHEN, QINGQU GUO, BO ZHANG, MUXING KANG, QIUPING XIE and YULIAN WU \\ Department of Surgery, Second Affiliated Hospital, College of Medicine, \\ Zhejiang University, Hangzhou, Zhejiang 310009, P.R. China
}

Received March 5, 2012; Accepted June 13, 2012

DOI: $10.3892 / \mathrm{ol} .2012 .783$

\begin{abstract}
Bufalin, an active component of the Chinese medicine chan'su, has been reported to have an inhibitory effect on the growth of various types of cancer cells. In the present study, we investigated whether gemcitabine combined with bufalin enhanced the antitumor efficacy in pancreatic cancer. Three pancreatic cancer cell lines (Bxpc-3, Mia PaCa-2 and Panc-1) were treated with gemcitabine and/or bufalin in vitro. The combination treatment demonstrated greater inhibition of cellular growth and apoptosis. The activity of apoptosis signal-regulating kinase 1 (ASK1)/JNK was upregulated in gemcitabine-induced apoptosis when combined with bufalin. We also observed that tumor growth was significantly inhibited by the combination therapy in a tumor-bearing mouse model, and upregulation of ASK1 activity was validated by immunohistochemical staining. These results suggest that bufalin may be a potential chemotherapeutic agent for pancreatic cancer, which could enhance the antitumor efficacy of gemcitabine when used in combination, possibly through the activation of ASK1/JNK.
\end{abstract}

\section{Introduction}

Pancreatic cancer is one of the most devastating malignant tumors and the fifth most common cause of cancer-related mortality in developed countries (1). Due to the difficulties of early diagnosis and highly aggressive behavior (2), 85\% of patients already have local infiltration or metastasis at the time of diagnosis. Less than $20 \%$ of patients have the option of radical tumor resection following the initial diagnosis (3). Thus, the 5-year survival rate of patients with pancreatic cancer is less than 5\% (4,5). Apart from surgery, chemotherapy is an essential auxiliary treatment for the management of advanced pancreatic cancer. As the first-line chemotherapy drug for pancreatic cancer, gemcitabine has been widely used in the clinic (6).

Correspondence to: Professor Yulian Wu, Department of Surgery, Second Affiliated Hospital, College of Medicine, Zhejiang University, 88 Jiefang Road, Hangzhou, Zhejiang 310009, P.R. China E-mail:wuyulian@medmail.com.cn

Key words: bufalin, pancreatic cancer, gemcitabine, apoptosis signal-regulating kinase 1 , c-Jun N-terminal protein kinase
However, due to a high degree of acquired and inherent resistance to pancreatic cancer chemotherapy (7), up to $20 \%$ of pancreatic cancer patients show no obvious effect following treatment with gemcitabine monotherapy (8). Thus, combined therapy with gemcitabine has gained considerable attention in the attempt to improve the outcome of pancreatic cancer (9).

Bufalin, an significant active component of the Chinese medicine chan'su (10), has widely demonstrated antitumor effects on human leukemia as well as ovarian, prostate and lung cancer (10-13). A possible mechanism of the antitumor effect of bufalin may be through the regulation of the MAPK signaling pathway and activation of a variety of transcription factors and protein kinases (14-16). It has been demonstrated that bufalin induces apoptosis in these cells via the activation of AP-1, the c-Jun N-terminal protein kinase (JNK), as well as by the induction of bcl-2 and the inhibition of protein kinase A. However, the effect of bufalin on pancreatic cancer cells has not yet been thoroughly evaluated.

Apoptosis signal-regulating kinase 1 (ASK1), also known as mitogen-activated protein kinase kinase kinase 5 (MAP3K5), a member of the MAPK family, is a serine/ threonine protein kinase which is an upstream activator of JNK and regulates diverse cellular responses. Previous studies have demonstrated that ASK1 participates in cell differentiation and apoptosis (14). The suppression of ASK1 may provide a general mechanism for cell survival, and overexpression of ASK1 is sufficient to cause apoptosis induced by reactive oxygen species (ROS) in a number of cell lines through many mitochondrial-dependent apoptotic stimuli including certain chemotherapeutic agents.

In this study, we investigated whether and how ASK1 becomes activated during cell death induced by bufalin. We found that bufalin interacts with and positively regulates ASK1 under various cell death conditions. Additionally, we investigated the synergistic effect on pancreatic cancer cell apoptosis induced by gemcitabine combined with bufalin. These findings suggest that ASK1 plays an important role in bufalin-mediated cell death and may enhance the antitumor effect of gemcitabine.

\section{Materials and methods}

Cell culture and reagents. Three human pancreatic cancer cell lines (Bxpc-3, Mia PaCa-2 and Panc-1) were purchased 
from American Type Culture Collection (ATCC, Rockville, MD, USA). The Mia PaCa-2 and Panc-1 cells were cultured in Dulbecco's modified Eagle's medium (DMEM; Gibco, Rockville, MD, USA) supplemented with $10 \%$ fetal bovine serum, $100 \mathrm{U} / \mathrm{ml}$ penicillin $\mathrm{G}$ and $100 \mathrm{U} / \mathrm{ml}$ streptomycin. The Bxpc-3 cells were cultured in RPMI-1640 (Gibco) containing supplements as above. All three cell lines were incubated at $37^{\circ} \mathrm{C}$ under $5 \% \mathrm{CO}_{2}$ in air. Bufalin, purchased from Sigma (St. Louis, MO, USA), was dissolved in dimethyl sulfoxide (DMSO) as a stock solution $(10 \mathrm{mM})$ and stored at $-20^{\circ} \mathrm{C}$. The culture media containing different concentrations of bufalin were all freshly prepared at the time of each experiment. The final concentration of DMSO was $<0.1 \%$. Gemcitabine was purchased from Ely Lilly (Bad Homburg, Germany) and dissolved in normal saline to make a $50 \mathrm{mg} / \mathrm{ml}$ stock solution.

Cell growth inhibition assay. The MTT [3-(4, 5-dimethylthiazol-2yl)-2, 5-diphenyltetrazolium bromide (Sigma) assay was used to assess the cellular viability. Briefly, cells were planted on a 96-well plate at a density of $5 \times 10^{3}$ cells per well and treated with drugs at different concentrations for 24, 48 and $72 \mathrm{~h}$. A total of $20 \mu \mathrm{l}$ MTT solution $[5 \mathrm{mg} / \mathrm{ml}$ in phosphatebuffered saline (PBS)] was added to each well, and further incubated for 3-5 h. Then, the culture medium was removed and the MTT formazan was dissolved in $150 \mu$ l DMSO. The plates were agitated for $10 \mathrm{~min}$, and absorbance was measured using an absorbance reader (BioTek ELx800, Winooski, VT, USA) at $490 \mathrm{~nm}$.

Flow cytometry and apoptosis detection. Cells were distributed on a 6-well plate at a density of $5 \times 10^{5}$ per well. After treatment with bufalin and/or gemicitabine for $48 \mathrm{~h}$, cells were harvested and washed with PBS three times. Then the degree of apoptosis was detected by Annexin V/FITC binding assay according to the manufacturer's instructions (BD Biosciences, Franklin Lakes, NJ, USA). The mixed solution was gently shaken and stored away from light at room temperature for $15 \mathrm{~min}$. The stained cells were analyzed directly by flow cytometry using Cell Quest software (BD Biosciences).

Transfection of siRNA. ASK1 siRNA was designed by GenePharma (Shanghai, China) with human ASK1 cDNA. The sequences designed against three separate regions starting from nucleotide 1258, 2025 or 2960 were: si-ASK1 1258, sense 5'-GGCAGCGAGUAGAUAAUAUTT-3' and antisense 5'-AUAUUAUCUACUCGCUGCCTT-3'; si-ASK1 2025, sense 5'-GUGGUUAGGUUUCCAGUAUTT-3' and antisense 5'-AUACUGGAAACCUAACCACTT-3'; si-ASK1 2960, sense 5'-GGGCUGUACAAUCAUUGAATT-3' and antisense 5'-UUCAAUGAUUGUACAGCCCTT-3'. A non-specific oligonucleotide served as the negative control. The cells plated in 6-well plates were transfected with $100 \mathrm{nM}$ siRNA or negative control siRNA using Lipofectamine ${ }^{\mathrm{TM}} 2000$ (Invitrogen, Carlsbad, CA, USA) following the manufacturer's instructions. Briefly, when cells reached $60-70 \%$ confluence, a mixture of Lipofectamine 2000 and OPTI-MEM medium (Invitrogen) was incubated for $5 \mathrm{~min}$, then incubated with siRNA for a further $30 \mathrm{~min}$ at room temperature to allow the complex formation. The transfection complex was added to each well ensuring distribution over the entire plate surface. The OPTI-MEM medium was replaced with DMEM at 4-6 $\mathrm{h}$ after transfection. The cells were incubated for $48 \mathrm{~h}$ prior to being harvested for further analysis.

Protein extraction and western blot analysis. Following treatment as described above, cells were washed with cold PBS and lysed in pre-chilled lysis buffer [1.0 mM ethylenediamineteraacetate (EDTA), $50 \mathrm{mM}$ Tris-HCL (pH 7.4), 1\% NP40, $0.1 \%$ SDS, $0.5 \%$ deoxycholate, $150 \mathrm{mM} \mathrm{NaCl}$ and $2 \%$ protease inhibitor cocktail]. Following centrifugation at $13,000 \mathrm{x} \mathrm{g}$ for $30 \mathrm{~min}$, the supernatant was collected and quantitated using the BCA protein assay (Pierce Biotechnology, Inc., Rockford, IL, USA). Total protein $(40 \mu \mathrm{g})$ was separated in 8-10\% SDS-polyacrylamide denaturing gels and transferred to polyvinylidene difluoride membranes. After blocking in TBST $(10 \mathrm{mM}$ Tris-HCL pH 7.4, $150 \mathrm{mM} \mathrm{NaCl}$ and $0.1 \%$ Tween-20) with 5\% non-fat milk for $1 \mathrm{~h}$, the membranes were incubated with primary antibodies overnight at $4^{\circ} \mathrm{C}$, followed by horseradish peroxidase-conjugated secondary antibodies. Immunoblotting for bcl-2, cleaved caspase-3, ASK1 (Cell Signaling Technology, Inc., Danvers, MA, USA), JNK and p-JNK (Santa Cruz Biotechnology, Inc., Santa Cruz, CA, USA) was performed. Immunoreactive bands were visualized by enhanced chemiluminescence (Amersham Biosciences Biotech, Piscataway, NJ, USA).

Animal experiments. Four-week-old male nu/nu mice were purchased from the First Affiliated Hospital of Zhejiang University. All animal experiments were approved by the Laboratory Animal Regulations of the Ministry of Science and Technology of China. Each mouse was subcutaneously injected with $6 \times 10^{6} \mathrm{Mia} \mathrm{PaCa}-2$ cells in the back. Treatment was started when the subcutaneous tumors reached a minimum size of $100 \mathrm{~mm}^{3}$. The mice were randomly divided into four treatment groups: a) vehicle alone (control); b) bufalin $(0.1 \mathrm{mg} / \mathrm{kg}$, for 10 days $) ; \mathrm{c})$ gemcitabine $(125 \mathrm{mg} / \mathrm{kg}$, three times/week for 2 weeks); d) bufalin and gemcitabine in combination. Each group consisted of six animals. The dose of $125 \mathrm{mg} / \mathrm{kg}$ twice a week for gemcitabine has been shown to be efficient in another pancreatic cancer xenograft model (17). To evaluate the tolerable therapeutic dose of bufalin in this animal model, we performed a preliminary dose-response experiment. Four times weekly i.p. injections of 1, 0.5, $0.2,0.1$ and $0.05 \mathrm{mg} / \mathrm{kg}$ bufalin were performed. Then, we determined the bufalin dose $(0.1 \mathrm{mg} / \mathrm{kg})$ for this study. The tumor size was measured every four days. The volume was calculated using the formula: volume $=\left(\right.$ length $\mathrm{x}$ width $\left.{ }^{2}\right) / 2$. One month after the treatment, the xenografts were excised and stocked in $10 \%$ formalin.

Immunohistochemical examination. Tissue sections $(4 \mu \mathrm{m})$ were prepared using a microtome and placed on glass slides. For immunohistochemical examination, endogenous peroxidase was blocked in $3 \% \mathrm{H}_{2} \mathrm{O}_{2}$ solution. Sections were incubated at $4^{\circ} \mathrm{C}$ with primary antibodies overnight. Following the removal of unbound antibodies, sections were incubated with biotinylated anti-mouse or anti-rabbit antibodies for $1 \mathrm{~h}$, and then incubated with horseradish peroxidase complex for $10 \mathrm{~min}$, followed by counterstaining with hematoxylin, dehy- 
A

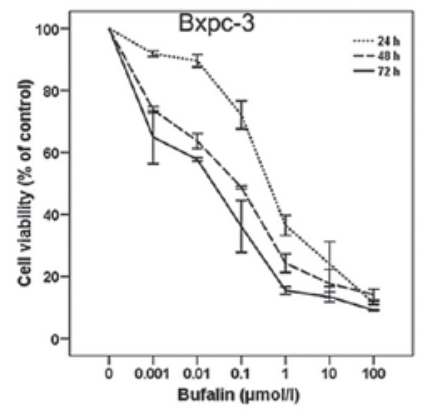

B

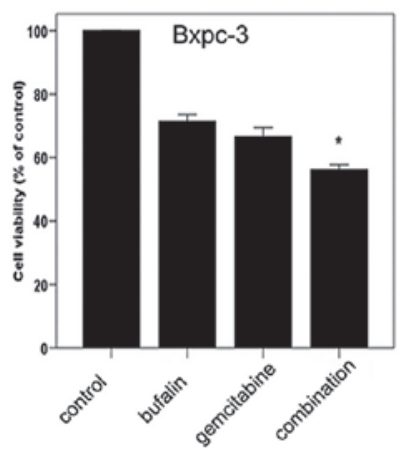

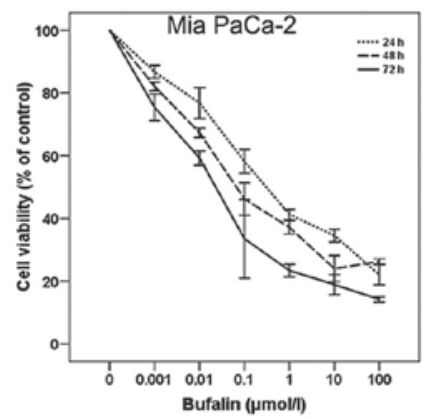

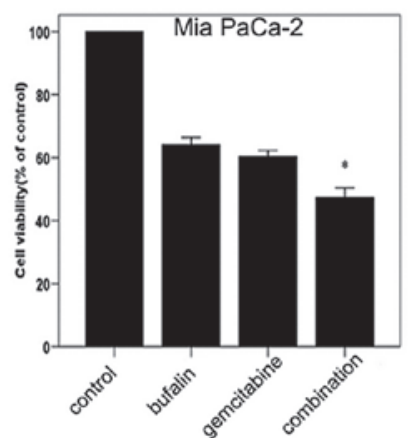

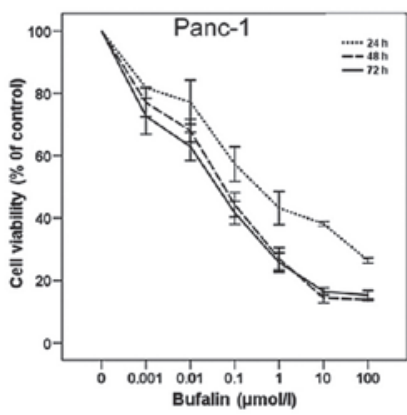

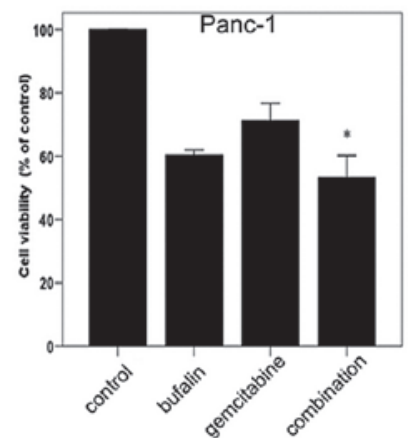

Figure 1. Bufalin inhibits the proliferation of three pancreatic cancer cell lines. (A) Bxpc-3, Mia PaCa-2 and Panc-1 cells were treated with bufalin for 24, 48 and $72 \mathrm{~h}$. The viability was assessed by MTT. Bufalin was shown to induce a dose- and time-dependent loss in all three cell lines. (B) Cells treated with bufalin $(0.01 \mu \mathrm{M})$ and/or gemcitabine [Bxpc-3 $(0.5 \mu \mathrm{g} / \mathrm{ml})$, Panc-1 and Mia PaCa-2 $(5 \mu \mathrm{g} / \mathrm{ml})$, respectively] for $48 \mathrm{~h}$. Compared with treatment of bufalin or gemcitabine alone, the combination group was statistically different ( $\mathrm{P}<0.05)$. Columns, mean of three experiments; bars, standard error (SE); ${ }^{*} \mathrm{P}<0.05$ compared with respective group.

dration and mounting. Sections without primary antibodies were used as negative controls for immunostaining. Random images obtained from each of the four groups were captured and analyzed at x400 magnification. The primary antibodies of anti-human Ki-67 and ASK1 were purchased from Cell Signaling Technology.

Statistical analysis. Results are presented as the mean \pm standard error (SE), and all experiments were performed three times independently. The one-way analysis of variance (ANOVA) and the two-tailed Student's t-test for unpaired samples were used to determine the statistical significance using SPSS 15.0. $\mathrm{P}<0.05$ was considered to indicate a statistically significant result.

\section{Results}

Bufalin potentiates growth inhibition induced by gemcitabine in pancreatic cancer cell lines. MTT assay was used to examine the cell growth inhibition efficacy of bufalin on three pancreatic cancer cell lines (Bxpc-3, Mia PaCa-2 and Panc-1). Cells were treated with bufalin at different concentrations (0-100 $\mu \mathrm{M})$ for 24,48 and $72 \mathrm{~h}$. The results demonstrated that bufalin inhibited the growth of all three cell lines in a dose- and time-dependent manner (Fig. 1A). We subsequently investigated the effect of the combination with bufalin and gemcitabine on cell viability. Pancreatic cancer cell lines were treated with bufalin $(0.01 \mu \mathrm{M})$ and/or gemcitabine (Bxpc-3, $0.5 \mu \mathrm{g} / \mathrm{ml}$; Mia PaCa-2 and Panc-1, $5 \mu \mathrm{g} / \mathrm{ml}$ ) for $48 \mathrm{~h}$ (18). Our results showed that the combination treatment with bufalin and gemcitabine inhibited the growth of all three cell lines more than either bufalin or gemcitabine used alone (Fig. 1B).

Bufalin enhances the induction of apoptosis by gemcitabine in pancreatic cancer cells. We investigated whether bufalin was capable of enhancing gemcitabine-induced apoptosis using flow cytometry analysis. The three pancreatic cancer cell lines were treated with different doses of drugs for $48 \mathrm{~h}$ in the same way as for the MTT assay. Exposure to bufalin $(0.01 \mu \mathrm{M})$ induced apoptosis by up to $7.8 \%$ in Bxpc-3, $11.5 \%$ in Mia Paca-21 and 7\% in Panc-1. Exposure to gemcitabine $(0.5$ or $5 \mu \mathrm{g} / \mathrm{ml})$ in combination with bufalin $(0.01 \mu \mathrm{M})$ for $48 \mathrm{~h}$ induced apoptosis by up to $16.8 \%$ in Bxpc-3, $21.8 \%$ in Mia Paca-2 and $17.4 \%$ in Panc-1 (Fig. 2A). To further examine the effect of inducing apoptosis by combination therapy, the expression of apoptosis-related proteins (bcl-2 and cleaved caspase-3) was evaluated. As shown in Fig. 2B, the combined treatment of pancreatic cancer cells with bufalin and gemcitabine significantly decreased the expression of bcl-2. Conversely, the expression of cleaved caspase-3 was significantly upregulated in the combination group compared with the control group and the bufalin and gemcitabine alone groups (Fig. 2B).

Bufalin upregulates the expression of ASK1 in three pancreatic cancer cell lines. To further investigate the apoptosis induced by bufalin, we evaluated the expression of ASK1 in the three pancreatic cancer cell lines. As shown in Fig. 3A, 
A
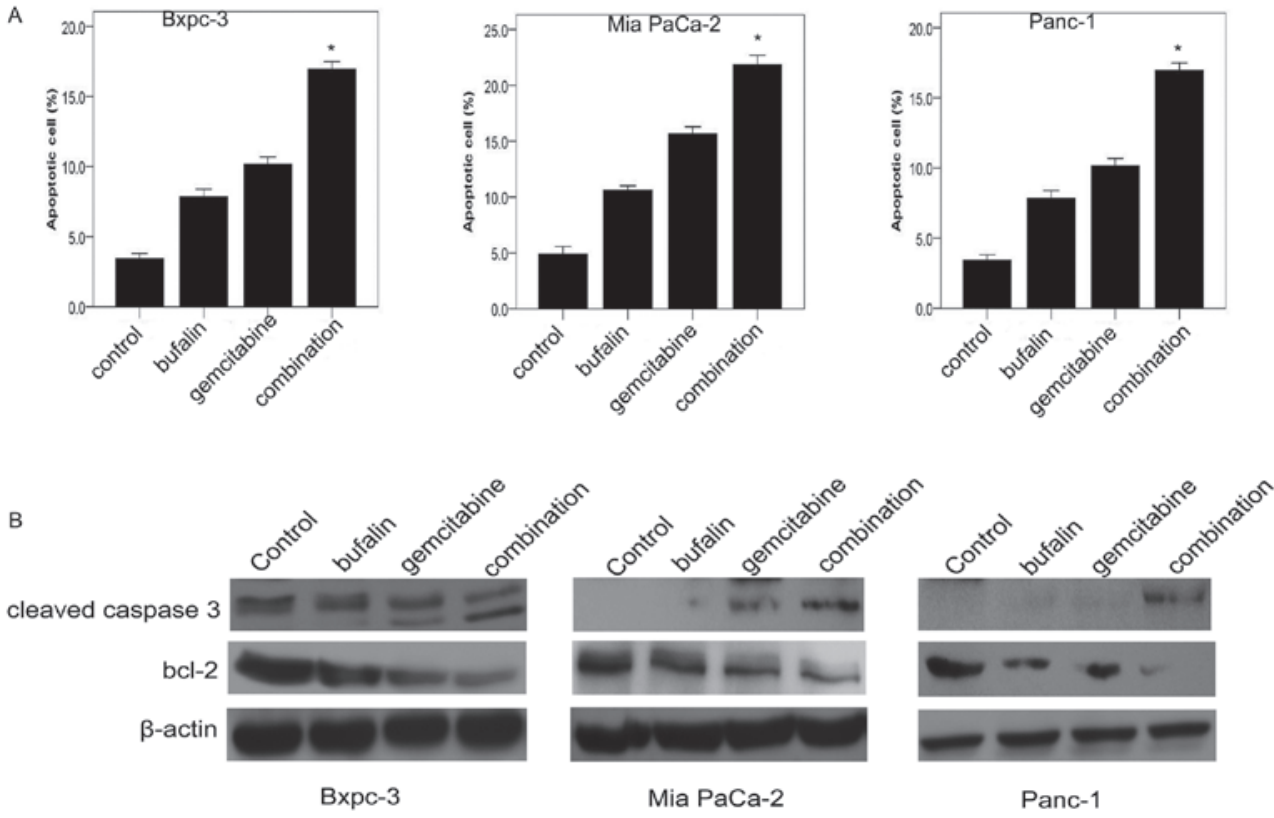

Figure 2. Potentiation of gemcitabine-induced apoptosis by bufalin in three pancreatic cancer cell lines. (A) Bxpc-3, Mia PaCa-2 and Panc-1 cells were treated with bufalin and/or gemcitabine for $48 \mathrm{~h}$, and then analyzed by flow cytometry. A significant difference was observed with the combination treatment compared with bufalin or gemcitabine treatment alone.(B) Protein of cleaved caspase- 3 and bcl- 2 in the three pancreatic cancer cell lines was extracted and analyzed by western blot analysis. Cleaved caspase- 3 was activated and bcl- 2 was downregulated. $\beta$-actin served as the internal control. Data are the results of three independent experiments.

\begin{tabular}{|c|c|c|c|c|}
\hline bufalin $(\mu \mathrm{M})$ & 0 & 0.001 & 0.01 & 0.1 \\
\hline \multicolumn{5}{|l|}{ ASK 1} \\
\hline \multicolumn{5}{|l|}{$\beta$-actin } \\
\hline \multicolumn{5}{|c|}{ Bхpc-3 } \\
\hline time $(h)$ & 0 & 12 & 24 & 48 \\
\hline ASK 1 & 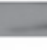 & -1 & & \\
\hline$\beta$-actin & & & & \\
\hline
\end{tabular}

Mia PaCa-2

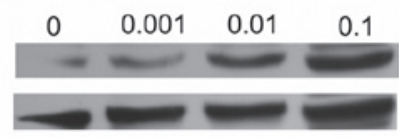

Mia PaCa-2

\begin{tabular}{llll}
0 & 12 & 24 & 48 \\
\hline & & & \\
\hline & &
\end{tabular}

Panc-1

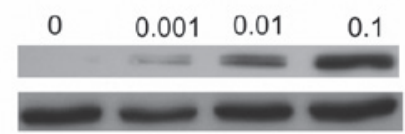

Panc-1

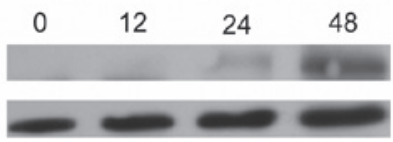

Figure 3. The role of ASK1 in the apoptotic pathway induced by bufalin in Bxpc-3, Mia PaCa-2 and Panc-1 cells. (A) Dose response of bufalin upregulated the expression of ASK1 in Mia PaCa-2 cells. Total proteins were prepared from cells after incubation with different concentrations of bufalin for $48 \mathrm{~h}$ as described in Materials and methods. (B) Time course of upregulation of ASK1 induced by bufalin in Bxpc-3, Mia PaCa-2 and Panc-1 cells. The cells were incubated with $0.01 \mu \mathrm{M}$ bufalin and the total protein was extracted for evaluation of ASK1 induction.

relative to the control, treatment with bufalin induced a dose-dependent increasing expression of ASK1 from 0.001 to $0.1 \mu \mathrm{M}$ in the three pancreatic cancer cell lines. However, bufalin $(0.001 \mu \mathrm{M})$ treatment induced a higher ASK1 level in Bxpc-3 and Mia PaCa-2 cells than in Panc-1 cells (Fig. 3A). When cells were treated with bufalin at a dose of $0.01 \mu \mathrm{M}$, the expression of ASK1 protein did not differ significantly among the groups. In addition, ASK1 expression was analyzed at a different treatment time with $0.01 \mu \mathrm{M}$ bufalin. A bufalininduced time-dependent activation of ASK1 expression was observed in Bxpc-3, Mia PaCa-2 and Panc-1 cells (Fig. 3B). However, treatment with bufalin $(0.01 \mu \mathrm{M})$ did not induce ASK1 expression until after $12 \mathrm{~h}$ in Panc-1 cells. The results also reveal that ASK1 expression was significantly upregulated after $48 \mathrm{~h}$ of treatment in the three pancreatic cancer cell lines (Fig. 3B). The Mia PaCa-2 cells treated with bufalin at $0.01 \mu \mathrm{M}$ for $48 \mathrm{~h}$ were selected for our next experiment.

Bufalin increased ASK1 expression induced by gemcitabine. We analyzed whether gemcitabine could induce ASK1 expression and whether upregulation of ASK1 by bufalin could eliminate chemoresistance in $\mathrm{Mia} \mathrm{PaCa}-2$ cells, resulting in more marked gemcitabine-induced apoptosis. We found that the level of ASK1 protein was increased when Mia PaCa-2 cells were treated with gemcitabine for $48 \mathrm{~h}$ (Fig. 4A). We also tested whether the combined treatment with bufalin for $48 \mathrm{~h}$ could abrogate gemcitabine-induced ASK1 protein expression levels. Our results revealed that ASK1 expression induced by gemcitabine increased in the bufalin combination treatment (Fig. 4A). 

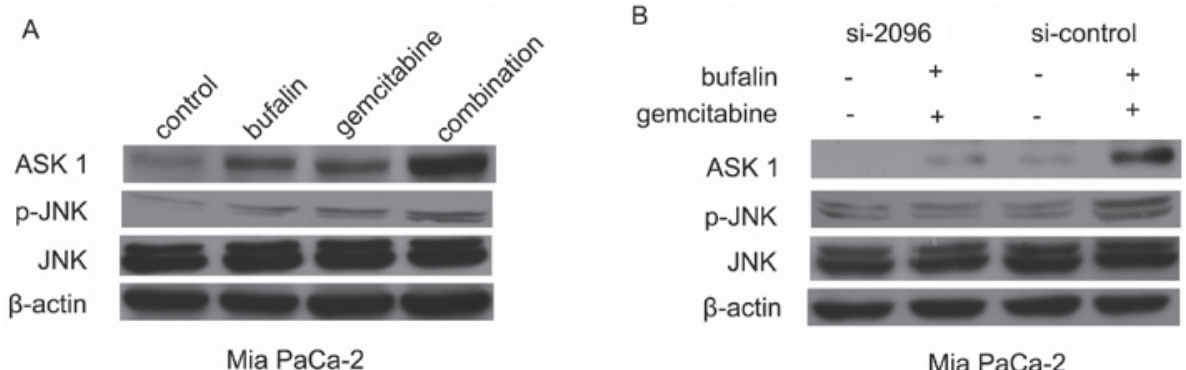

Figure 4. Bufalin inhibited the ASK1/JNK signaling pathway in Mia PaCa-2 cells. (A) Mia PaCa-2 cells were treated with gemcitabine (5 mg/ml), bufalin $(0.01 \mu \mathrm{M})$ and the combination for $48 \mathrm{~h}$. The expression of ASK1, p-JNK and JNK were investigated. (B) Mia PaCa-2 cells were transfected with various siRNAs as indicated, and the expression of ASK1 and p-JNK was determined after treatment with bufalin and gemcitabine for $48 \mathrm{~h}$. A reduced level of p-JNK was detected by western blot analysis. $\beta$-actin protein served as the loading control.
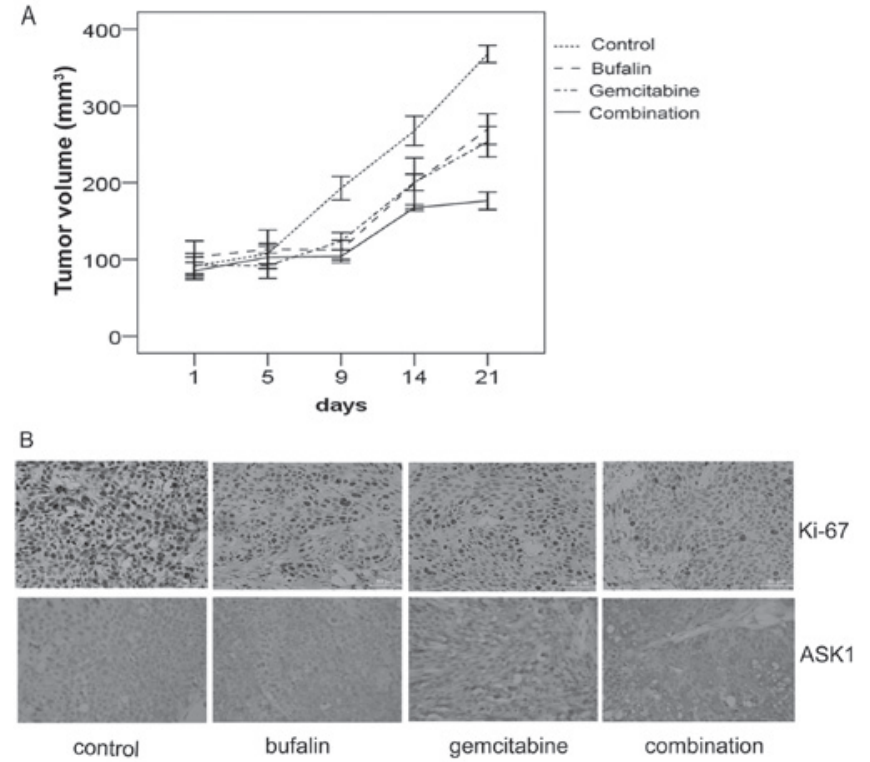

Figure 5. The combination treatment of gemcitabine with bufalin inhibited tumor growth in xenograft model. (A) The tumor volume in pancreatic cancer xenograft model after treatment. There was a statistically significant difference between the combination treatment group and the control group $(\mathrm{P}<0.05)$. (B) The expression of Ki-67 was decreased and ASK1 was increased in the combination group.

Bufalin induced apoptosis in pancreatic cancer cells, possibly via ASK1/JNK pathway. ASK1 activation is a pivotal mechanism in a broad variety of cell apoptosis. To explore whether the ASK1/JNK pathway contributes to bufalin-induced apoptosis in pancreatic cancer cells, the expression of ASK1 and p-JNK proteins were investigated by western blot analysis. The expression of ASK1 was upregulated with the combined treatment in Mia PaCa-2 cells. Furthermore, such effect of p-JNK was also detected in Mia PaCa-2 cell treatment by bufalin with or without gemcitabine (Fig. 4A). Next, ASK1 siRNA was transfected into cancer cells. The expression of ASK1 in cells transfected with si-2096 decreased to $20 \%$ of that of the si-control. Immunoblotting was performed to examine the expression of $\mathrm{p}-\mathrm{JNK}$ in the treatment group transfected with si-2096 or with si-control in Mia PaCa-2 cells (Fig. 4B). No difference in expression was observed with total JNK, demonstrating that bufalin and gemcitabine have no effect on the total JNK. The expression of p-JNK was signifi- cantly decreased following the combined treatment in si-2096 Mia PaCa-2 cells, suggesting that the increased level of p-JNK could be downregulated by si-ASK1 with the combination treatment in pancreatic cancer cells (Fig. 4B).

Bufalin potentiates the antitumor effect of gemcitabine in vivo. We used the Mia PaCa-2 subcutaneous xenograft as an in vivo model. Following the combination treatment with gemcitabine and bufalin for two weeks, the tumor volume was significantly reduced compared with that observed in the groups treated with control, gemcitabine and bufalin alone (Fig. 5A). Ki-67 nuclear antigen is used to determine cell proliferation activity, and is a key indicator of prognosis for certain malignant tumors. It was found to be significantly decreased in the combination group. In addition, ASK1 was found to be significantly upregulated in the combination group (Fig. 5B).

\section{Discussion}

The survival rate of patients with pancreatic carcinoma is rather poor, mainly because the disease is frequently diagnosed at an advanced stage, and is characterized by a chemo-resistant phenotype (19). Several studies have shown that cytotoxic agents block tumorigenic cascade activation during cancer initiation and progression (20). In particular, these targeted therapies could be used in combination with current clinical chemotherapeutic drug regimens such as gemcitabine and/or 5-fluorouracil (5-FU) to overcome drug resistance and improve the efficacy of treatments for patients with locally advanced or metastatic pancreatic cancer (21). In the present study, we used bufalin in combination with gemcitabine to estimate its efficacy against pancreatic cancer cells.

Bufalin has been reported to play a critical role in cancer cell apoptosis and differentiation, in ovarian and prostate cancer $(11,12)$, with little toxic effect on normal cells at low doses (11). Bufalin also induces the generation of ROS in lung and colon cancer $(22,23)$. However, the role of bufalin in pancreatic cancer cell lines has not been investigated. In our study, a dose- and time-dependent growth inhibition was observed in the MTT assay when cells were treated with bufalin. Next, we examined whether bufalin enhanced the sensitivity of gemcitabine in pancreatic cancer cell lines. The results revealed that the combination treatment with gemcitabine and bufalin enhanced tumor cell growth inhibition compared with either agent alone. 
By flow cytometry analysis, potentiation of gemcitabineinduced apoptosis by bufalin in pancreatic cancer cells was also observed. In accordance with the results mentioned above, the potentiation of gemcitabine-induced apoptosis by bufalin in pancreatic cancer cells was validated by enhancing cleaved caspase- 3 activity and inhibiting bcl- 2 protein. These results may be significant in understanding the role of bufalin in the gemcitabine-induced cell apoptosis of pancreatic cancer.

It is well-known that the bcl-2 protein is an anti-apoptotic factor, which confers resistance to gemcitabine in pancreatic cancer cells. SiRNA-mediated silencing of bcl-2 enhances gemcitabine sensitivity in human pancreatic cancer cells (24). A previous study has reported that decreasing bcl-2 levels were associated with bufalin-induced apoptosis (10). In our study, the level of bcl-2 was downregulated in the combination group compared with bufalin or gemcitabine used alone, suggesting that bufalin may enhance the effect of gemcitabine by downregulating the levels of bcl-2 in pancreatic cancer cells.

ASK1 is a ROS-sensitive protein, which is involved in the activation of AP-1, Rac1, cdc2 kinase and JNK, as well as the inhibition of protein kinases A and C (25-27). It constitutes a pivotal signaling pathway in cytokine- and stress-induced apoptosis $(28,29)$. Activation of the JNK family is involved in various physiological and pathological processes, including cell apoptosis, inflammatory response and cytokine production (30-32). JNKs are activated following dual phosphorylation of threonine and tyrosine specifically by MKK4 and MKK7 (33). Overexpression of ASK1 may induce cytochrome $\mathrm{c}$ release from the mitochondria and activate caspase-9 and caspase-3 (28). Furthermore, Yu et al reported that the ASK $1 / \mathrm{JNK}$ signaling cascade contributed to denbinobin-induced apoptosis in A549 cells (34). Yamamoto et al demonstrated that ASK1-mediated JNK activation phosphorylated bcl-2, leading to a reduction in its anti-apoptotic activity (35). However, whether the ASK1/JNK signaling pathway participates in bufalin-induced apoptosis in pancreatic cancer has not previously been demonstrated. In this study, we found that treatment of pancreatic cancer cells with bufalin caused the activation of ASK1 and p-JNK. Furthermore, treatment with the combination therapy significantly increased the expression of ASK1/JNK in Mia PaCa-2 cells. When ASK1 was knocked down, the level of p-JNK was decreased in cells with combined treatment of bufalin and gemcitabine. These results suggest that bufalin may, at least partially, enhance the antitumor effect of gemcitabine in pancreatic cancer by activating ASK1 to induce JNK activation, which ultimately leads to bcl-2 expression in pancreatic cancer cells. In the tumor-bearing animal model, the results were replicated in vitro. The final tumor volumes in the combination group were significantly reduced compared with the control group and the gemcitabine alone group. The expression of Ki-67 was notably reduced in tumor tissue treated with the combination therapy. More importantly, the expression of ASK1 increased in tumor tissues with the combined treatment.

In conclusion, the results from the present study demonstrated for the first time that it was possible to enhance the chemo-sensitivity of pancreatic cancer cells through treatment with bufalin. Apoptosis may be mediated by the upregulation of the ASK1/JNK pathway, which eventually induces bcl-2 expression in human pancreatic cancer. Our results provide a mechanism linking bufalin and apoptosis kinase ASK1 and provide support for the development of therapeutic strategies to overcome the resistance to gemcitabine in pancreatic cancer chemotherapy.

\section{Acknowledgements}

This study was supported by grants from the National Natural Science Foundation of China (nos. 30872531 and 81001094), and the Ministry of Science and Technology of the People's Republic of China (no. 2007AA02Z476).

\section{References}

1. Jemal A, Siegel R, Ward E, et al: Cancer statistics, 2006. CA Cancer J Clin 56: 106-130, 2006.

2. Shah AN, Summy JM, Zhang J, Park SI, Parikh NU and Gallick GE: Development and characterization of gemcitabineresistant pancreatic tumor cells. Ann Surg Oncol 14: 3629-3637, 2007.

3. Jemal A, Murray T, Samuels A, Ghafoor A, Ward E and Thun MJ: Cancer statistics, 2003. CA Cancer J Clin 53: 5-26, 2003.

4. Riall TS, Nealon WH, Goodwin JS, et al: Pancreatic cancer in the general population: Improvements in survival over the last decade. J Gastrointest Surg 10: 1212-1223; discussion 1223-1214, 2006.

5. Pearce HL and Alice Miller M: The evolution of cancer research and drug discovery at Lilly Research Laboratories. Adv Enzyme Regul 45: 229-255, 2005.

6. Shen Y, Cai M, Xia W, et al: FTY720, a synthetic compound from Isaria sinclairii, inhibits proliferation and induces apoptosis in pancreatic cancer cells. Cancer Lett 254: 288-297, 2007.

7. Lee SH, Ryu JK, Lee KY, et al: Enhanced anti-tumor effect of combination therapy with gemcitabine and apigenin in pancreatic cancer. Cancer Lett 259: 39-49, 2008.

8. Burris HA III, Moore MJ, Andersen J, et al: Improvements in survival and clinical benefit with gemcitabine as first-line therapy for patients with advanced pancreas cancer: a randomized trial. J Clin Oncol 15: 2403-2413, 1997.

9. Kimura K, Sawada T, Komatsu M, et al: Antitumor effect of trastuzumab for pancreatic cancer with high HER-2 expression and enhancement of effect by combined therapy with gemcitabine. Clin Cancer Res 12: 4925-4932, 2006

10. Watabe M, Kawazoe N, Masuda Y, Nakajo S and Nakaya K: Bcl-2 protein inhibits bufalin-induced apoptosis through inhibition of mitogen-activated protein kinase activation in human leukemia U937 cells. Cancer Res 57: 3097-3100, 1997.

11. Takai N, Ueda T, Nishida M, Nasu K and Narahara H: Bufalin induces growth inhibition, cell cycle arrest and apoptosis in human endometrial and ovarian cancer cells. Int J Mol Med 21: 637-643, 2008

12. Yu CH, Kan SF, Pu HF, Jea Chien E and Wang PS: Apoptotic signaling in bufalin- and cinobufagin-treated androgendependent and -independent human prostate cancer cells. Cancer Sci 99: 2467-2476, 2008.

13. Nasu K, Nishida M, Ueda T, et al: Bufalin induces apoptosis and the G0/G1 cell cycle arrest of endometriotic stromal cells: a promising agent for the treatment of endometriosis. Mol Hum Reprod 11: 817-823, 2005

14. Kuo CT, Chen BC, Yu CC, et al: Apoptosis signal-regulating kinase 1 mediates denbinobin-induced apoptosis in human lung adenocarcinoma cells. J Biomed Sci 16: 43, 2009.

15. Schoner W and Scheiner-Bobis G: Endogenous and exogenous cardiac glycosides: their roles in hypertension, salt metabolism, and cell growth. Am J Physiol Cell Physiol 293: C509-536, 2007.

16. Amano Y, Cho Y, Matsunawa M,Komiyama K and Makishima M: Increased nuclear expression and transactivation of vitamin D receptor by the cardiotonic steroid bufalin in human myeloid leukemia cells. J Steroid Biochem Mol Biol 114: 144-151, 2009.

17. Tang ZY, Wu YL, Gao SL and Shen HW: Effects of the proteasome inhibitor bortezomib on gene expression profiles of pancreatic cancer cells. J Surg Res 145: 111-123, 2008.

18. Guo Q, Chen Y, Zhang B, Kang M, Xie Q and Wu Y: Potentiation of the effect of gemcitabine by emodin in pancreatic cancer is associated with survivin inhibition. Biochem Pharmacol 77: 1674-1683, 2009 
19. Kornmann M, Beger HG and Link KH: Chemosensitivity testing and test-directed chemotherapy in human pancreatic cancer. Recent Results Cancer Res 161: 180-195, 2003.

20. Zalatnai A and Molnar J: Review. Molecular background of chemoresistance in pancreatic cancer. In Vivo 21: 339-347, 2007.

21. Mimeault M, Hauke R and Batra SK: Recent advances on the molecular mechanisms involved in the drug resistance of cancer cells and novel targeting therapies. Clin Pharmacol Ther 83: 673-691, 2008

22. Sun L, Chen T, Wang X, Chen Y and Wei X: Bufalin induces reactive oxygen species dependent bax translocation and apoptosis in ASTC-a-1 cells. Evid Based Complement Alternat Med 2009.

23. Xie CM, Chan WY, Yu S, Zhao J and Cheng CH: Bufalin induces autophagy-mediated cell death in human colon cancer cells through reactive oxygen species generation and JNK activation. Free Radic Biol Med 51: 1365-1375, 2011.

24. Okamoto K, Ocker M, Neureiter D, et al: bcl-2-specific siRNAs restore gemcitabine sensitivity in human pancreatic cancer cells. J Cell Mol Med 11: 349-361, 2007.

25. Kurosawa M, Numazawa S, Tani Y and Yoshida T: ERK signaling mediates the induction of inflammatory cytokines by bufalin in human monocytic cells. Am J Physiol Cell Physiol 278: C500-508, 2000

26. Kawazoe N, Watabe M, Masuda Y, Nakajo S and Nakaya K Tiam1 is involved in the regulation of bufalin-induced apoptosis in human leukemia cells. Oncogene 18: 2413-2421, 1999.

27. Kurosawa M, Tani Y, Nishimura S, Numazawa S and Yoshida T: Distinct PKC isozymes regulate bufalin-induced differentiation and apoptosis in human monocytic cells. Am J Physiol Cell Physiol 280: C459-C464, 2001.
28. Hatai T, Matsuzawa A, Inoshita S, et al: Execution of apoptosis signal-regulating kinase 1 (ASK1)-induced apoptosis by the mitochondria-dependent caspase activation. J Biol Chem 275: 26576-26581, 2000.

29. Ichijo H, Nishida E, Irie K, et al: Induction of apoptosis by ASK1, a mammalian MAPKKK that activates SAPK/JNK and p38 signaling pathways. Science 275: 90-94, 1997.

30. Numazawa S, Shinoki MA, Ito H, Yoshida T and Kuroiwa Y: Involvement of $\mathrm{Na}+, \mathrm{K}(+)$-ATPase inhibition in $\mathrm{K} 562$ cell differentiation induced by bufalin. J Cell Physiol 160: 113-120, 1994.

31. Jing Y, Watabe M, Hashimoto S, Nakajo S and Nakaya K: Cell cycle arrest and protein kinase modulating effect of bufalin on human leukemia ML1 cells. Anticancer Res 14: 1193-1198, 1994

32. Masuda Y, Kawazoe N, Nakajo S, Yoshida T, Kuroiwa Y and Nakaya K: Bufalin induces apoptosis and influences the expression of apoptosis-related genes in human leukemia cells. Leuk Res 19: 549-556, 1995.

33. Su F, Li H, Yan C, Jia B, Zhang Y and Chen X: Depleting MEKK1 expression inhibits the ability of invasion and migration of human pancreatic cancer cells. J Cancer Res Clin Oncol 135: 1655-1663, 2009.

34. Yu CC, Hsu MJ, Kuo ML, et al: Thrombin-induced connective tissue growth factor expression in human lung fibroblasts requires the ASK1/JNK/AP-1 pathway. J Immunol 182: 7916-7927, 2009.

35. Yamamoto K, Ichijo H and Korsmeyer SJ: BCL-2 is phosphorylated and inactivated by an ASK1/Jun N-terminal protein kinase pathway normally activated at G(2)/M. Mol Cell Biol 19: 8469-8478, 1999. 